\title{
Rechercher la vie parfaite? Enjeux autour du handicap
}

\author{
J. Martin ${ }^{a}$
}

\footnotetext{
a Le Dr Martin est membre de la Commission nationale d'éthique.
}

Correspondance: Dr Jean Martin, privat-docent Médecin cantonal Service de la santé publique CH-1014 Lausanne
En août 2002 à Neuchâtel, l'un des quatre sites de l'Exposition nationale, la Commission nationale d'éthique pour la médecine humaine (CNE), nommée par le Conseil fédéral en juillet 2001, organisait des forums publics sur plusieurs thèmes. L'un d'eux, débattant $\mathrm{d}^{\prime}$ «Une vie parfaite, idéal ou cauchemar?», a donné lieu à des échanges tout à fait stimulants. Est-ce le but de la médecine, de la recherche voire des pouvoirs publics, de promouvoir une telle perfection? Rapidement, on s'est mis d'accord sur le fait que sa poursuite n'était pas réaliste ni même raisonnable. Les démarches absolues courent toujours le risque d'être totalitaires, comme l'observation quotidienne de notre monde le démontre. Il a été relevé qu'il y avait même là une contradiction dans les termes: la vie ne saurait être parfaite, seule la mort l'est.

A l'évidence, la manière dont, avec une moindre ambition, on apprécie une vie réussie dépend beaucoup du cadre de référence culturel, des valeurs de la société dans laquelle on vit et des siennes propres. Aujourd'hui, marqués que nous sommes par l'esprit du temps des dernières décennies, nous oublions que la réussite d'une vie ne dépend pas fatalement d'un air jeune, d'un métier prestigieux, d'un poids civil ou politique, de l'argent qu'on gagne et qu'on dépense et de l'accumulation de biens divers. Chez nous aussi, certains mettent leurs priorités ailleurs: dans une vie utile à rendre les choses et circonstances autour de soi un peu meilleures, parfois véritablement au service de son prochain, ou dans une insertion familiale et sociale substantielle. On peut (comme l'artiste, l'artisan ou le berger sur son alpage) avoir comme impératif une activité qui satisfait même si elle n'est guère rémunératrice. Et même s'il est rare aujourd'hui de consacrer sa force et son existence à la spiritualité, la méditation ou la contemplation, on se souvient que, ailleurs plus qu'ici, des dizaines de milliers de personnes le font, pour toute leur vie ou durant certaines périodes, indifférentes aux conditions matérielles.

Lors du Forum de Neuchâtel de la Commission nationale d'éthique a été discutée la situation de ceux qui parmi nous vivent avec des limitations fonctionnelles et des troubles de santé de longue durée. Plusieurs personnes handicapées se sont exprimées. La notion de santé est tout à fait relative: il y des diabétiques ou des rhumatisants sévères dont on peut dire qu'ils mènent une vie heureuse, réussie (en santé!); et certains de nos concitoyens qui sont à l'aise matériellement, sont en pleine forme physique et ont un look d'acteur/actrice de cinéma traînent une existence qui ne les satisfait pas. On entend souvent des témoignages de personnes handicapées démontrant comment, dans les limites qui leur sont imposées, elles vivent (ont appris à vivre) pleinement. Bien que ce ne soit pas à un non-malade de le relever, l'histoire récente du sida a été illustrée par de nombreuses histoires de vie où, malgré un pronostic funeste, les personnes concernées ont dit connaître un épanouissement qu'elles n'imaginaient pas auparavant (ou que pour le moins elles n'avaient pas trouvé).

Ce qui a été soulevé dans la discussion, c'est la crainte que les progrès de la science et de la médecine n'amènent une discrimination accrue de ceux qui présentent un handicap, une différence. Je m'explique. Des mesures médicales permettent aujourd'hui de dépister un certain nombre d'affections et d'éviter la survenue d'enfants porteurs de maladies héréditaires ou congénitales (par le biais d'un traitement précoce efficace ou du moyen moins satisfaisant de l'interruption de grossesse). Le nombre de personnes «différentes» à cause d'une infirmité/malformation diminue actuellement; c'est un effet logique de l'amélioration de nos moyens et donc quelque chose qu'on attend des soignants. Mais le fait est que la plupart d'entre nous ont moins souvent l'occasion d'être en contact avec des congénères «différents» et par conséquent seront plus facilement mal à l'aise en les rencontrant: on ne saura pas comment se comporter, on évitera d'aborder la personne ou de lui parler, au propre et au figuré on fuira. Il n'est pas besoin d'être grand clerc pour voir que cela contribue à accroître l'isolement (physique, psychologique) des handicapés. Question éthique et sociale de grande importance. Ma fonction professionnelle me fait être au contact de soucis y relatifs de la part d'associations de personnes handicapées et de parents. 
Le débat a aussi porté sur les tensions possibles entre la liberté individuelle, que les dernières décennies ont vivement mis à l'avant de la scène, et l'intérêt général ou plus prosaïquement un certain bon sens collectif. Ainsi que sur les attentes que l'on a que des prestations soient assumées par les pouvoirs publics ou avec leur aide substantielle. A été évoquée l'histoire rapportée par les médias en 2002, aux Etats-Unis, de cette personne sourde qui a entrepris des démarches en vue d'être enceinte d'un enfant qui ait toutes les chances d'être sourd lui-même (en recherchant un donneur de sperme sourd). Au nom du fait, grosso modo, qu'on peut être sourd et épanoui, et dans la foulée de multiples mouvements récents affirmant qu'être porteur de telle caractéristique is beautiful (ou peut l'être). Il y a là une dimension sociétale, politique, qui mérite l'attention: il s'agit des attitudes qu'on adopte vis-à-vis de la dignité, des intérêts et des souhaits d'un groupe particulier, ce qu'on appelle - sans aucune connotation péjorative ici - une sous-culture (s'agissant de personnes sourdes par exemple, elles ne communiquent à l'évidence pas comme la plupart et une partie des potentialités culturelles usuelles - on pense à la musique - ne leur sont pas accessibles, mais elles peuvent en avoir d'autres).

Quant au principe, on peut comprendre une telle requête; quoique handicapées, ces personnes estiment avoir une vie riche; leur intention d'avoir une progéniture qui leur ressemble et vive des expériences comparables a sa logique. Cela étant, il est vraisemblable que beaucoup parmi nous auront de la difficulté à se rallier à l'intention en question. Ne peut-on pas, à l'inverse, penser que des enfants non sourds auront des potentialités/opportunités plus grandes, tout en vivant avec leurs parents sourds, en s'adaptant au handicap de ces derniers et en les rendant heureux (même sans partager toutes leurs expériences de vie)? Débattant de tels enjeux, il convient de manière approfondie de peser l'intérêt de l'enfant à naître.

Un autre point mérite d'être considéré, notamment du point de vue du serviteur public que je suis: on peut respecter la libre détermination de la femme sourde souhaitant un enfant sourd et qui, à titre privé, cherche un partenaire dont les caractéristiques augmentent les chances d'obtenir le résultat voulu. Une question toutefois est de savoir si les techniques médicales (procréation médicalement assistée), et le cas échéant les fonds publics consacrés à les mettre en œuvre, doivent être mis au service d'un tel projet de vie. Une vision très libertaire dira qu'il n'est pas admissible de poser des obstacles à la réalisation de la volonté d'une personne adulte douée de discernement; d'autres invoqueront un mandat général des pouvoirs publics de promouvoir une santé ou un bien-être définis selon l'appréciation usuelle de la population et des professionnels.

Enjeux éthiques et politiques stimulants et ardus. Inutile de dire que la Commission nationale d'éthique ne prétend pas en un tour de main apporter des réponses à la multiplicité de questions de cet ordre qui sont aujourd'hui posées, et à celles qui se poseront à l'avenir en nombre croissant. Mais le fait qu'il n'y ait pas de solutions aisées (aucune baguette magique!) ne saurait évidemment signifier qu'il n'est pas utile d'y réfléchir. Comme généralement en éthique, l'important n'est pas nécessairement de trouver une solution mais de poser les questions pertinentes, de voir quelles préoccupations particulières elles soulèvent et de considérer un éventail de marches à suivre possibles. Dans plusieurs registres nous avons un besoin urgent de consacrer plus de temps et d'énergie aux aspects philosophiques de ce que nous faisons ou ne faisons pas. 